\title{
Smartphone-Based Optical Fiber Sensor for the Assessment of a Fed-Batch Bioreactor ${ }^{+}$
}

\author{
Marco César Prado Soares *, Thiago Destri Cabral, Pedro Machado Lazari, \\ Matheus dos Santos Rodrigues, Gildo Santos Rodrigues and Eric Fujiwara \\ School of Mechanical Engineering, University of Campinas, São Paulo 13083-860, Brazil; \\ tdcabral@ifi.unicamp.br (T.D.C.); pedrolazarimachado@gmail.com (P.M.L.); \\ santos.r.math@gmail.com (M.d.S.R.); gildoesseerre@gmail.com (G.S.R.); fujiwara@fem.unicamp.br (E.F.) \\ * Correspondence: marcosoares.feq@gmail.com; Tel.: +55-19-3521-3337 \\ + Presented at the 7th Electronic Conference on Sensors and Applications, 15-30 November 2020; Available \\ online: https://ecsa-7.sciforum.net/.
}

Published: 15 November 2020

\begin{abstract}
Industry is currently in a period of great expansion, the so-called "Industry 4.0 ". It relies on the development of new sensor technologies for the generation of systems capable of collecting, distributing and delivering information. Particularly, on Chemical and Biochemical industries, the development of portable monitoring devices can improve many process parameters, like safety and productivity. In this work, the design of a smartphone-based optical fiber sensing platform for the online assessment of fed-batch fermentation systems is reported. The setup is comprised of a smartphone equipped with a 3D-printed case and an application for analyzing the pixel intensity, which is correlated to the broth refraction index (function of sucrose concentration). A sensitivity of 85.83 $\mathrm{RIU}^{-1}$ (refractive index unit) was verified, and the sensor performance was compared to a handheld refractometer and to model predictions. It showed to be a reliable, portable and low-cost instrument for online monitoring bioreactors, easily reproducible on-site by simply printing it.
\end{abstract}

Keywords: optical fiber sensors; bioreactor monitoring; fed-batch fermentation

\section{Introduction}

Nowadays, industry is in a period of great expansion. The so-called "Industry 4.0 " technologies apply novel mathematical and computer-based methods for the optimization and monitoring of systems, with social, economic, and environmental repercussions on the activities [1]. This new period relies on the development of new sensor technologies capable of collecting, distributing, and delivering information by themselves.

In Chemical and Biochemical industries, the increase on the data availability and of the portability of the monitoring devices has potential for enhancing safety, productivity, energy-use efficiency, environmental sustainability, product quality, and general process performance [2]. However, the analysis and evaluation of many chemical and biochemical agents (from pesticides to pharmacological drugs) is still based on high-performance liquid chromatography (HPLC), gas chromatography (GC), and coupled techniques like GC coupled to mass spectrometry (GC-MS) and enzyme-linked immunosorbent assay (ELISA). Such methods are sensitive, reliable, and precise, but demand expensive and bulky instrumentation, highly trained technicians, and procedures that require a long analysis time. The use of compact, real-time sensors, on the other hand, allows the monitoring, control, and screening of the best process conditions [2-4]. Optical fiber sensors, in particular, are very attractive for chemical assessment: they present biocompatibility, immunity to electromagnetic interference, and the fibers demonstrate chemical and thermal stability [5] and reduced fabrication costs, making the sensors suitable for mass-fabrication $[4,6]$. 
In this work, a portable smartphone-based optical fiber sensing platform is designed for the monitoring of fed-batch fermentation systems. The fed-batch mode was chosen for the study because most of the alcoholic fermentation industrial systems in Brazil operate with this methodology [7]. The results were validated by comparison with a handheld refractometer and with the mathematical model. The proposed platform is essentially different from other chemical and biochemical smartphone-based analytical tools already reported. They are usually based on electrochemical detection, applying the USB port of the phone for the evaluation of the current in an external circuit [8,9]. The optical monitoring smartphone systems are generally of higher complexity and involve high cost materials or are based on colorimetric detection by the camera [10]. Examples include a smartphone coupled to a commercial enzymatic chromogen-kit for detecting coliforms and bacteria in water [11]; and a bioluminescence colorimetric sensor that analyzes perfectly positioned spheroids of cells, which must be genetically modified for the emission of fluorescence, the physical parameter detected [12].

\section{Materials and Methods}

\subsection{Fermentation Monitoring and Modelling}

The monitoring and control of a fermentation process is focused on the maintenance of the adequate conditions for the microorganisms, and it is based on the evaluation of the cells' concentration X. Many of the traditional measurements (e.g., cell counting with Neubauer chamber; dry mass evaluation; and the surface plating method to determine viable cell number) are based on manual time-consuming procedures. Therefore, in most of the practical and industrial cases, the measurement and control are actually based on the quantification of a specific property, which may be either physical (e.g., variation of the medium's refractive index, viscosity, or electrical conductivity) or biochemical (concentration of proteins, carbohydrates, DNA or RNA, for example). These properties are posteriorly correlated to the concentration of cells by an appropriate model derived from the general fermentation reaction: $X+S \rightarrow P+(X+\Delta X)$, where a microorganism concentration $X$ consumes a substrate concentration S (e.g., sucrose, the "reactant" of cellular reactions), producing both a product concentration $\mathrm{P}$ (on yeast fermentation, it is usually ethanol) and an increase $\Delta X$ on the cellular concentration due to the cellular reproduction (it is an autocatalytic process) $[4,13,14]$. The mathematical kinetic models of cellular growth are also based on the definition of the specific cell growth rate $\mu=(1 / X)(d X / d t)$. One of these models is the Monod equation, given by $\mu=\mu_{m} S /\left(K_{M}+S\right)$, where $\mu_{m}$ is the maximum specific growth rate, and $K_{M}$ is the Monod constant [4]. The estimation of product formation and substrate uptake rates by cells can be performed with two supporting definitions: the specific rate of product formation $q_{p}=Y_{P / X} \mu+$ $m_{p}$, where $m_{p}$ is the product formation rate not associated to cellular growth, and $Y_{P / X}$ is the theoretical yield of product formation per cell reproduction; and the specific rate of substrate consumption $\mu_{s}=\left(1 / Y_{X / S}\right) \mu+m_{s}$, where $m_{s}$ is the substrate consumption rate associated to the metabolic activities, and $Y_{X / S}$ is the theoretical yield of cell reproduction per substrate uptake [4,15].

On fed-batch operation mode, the reaction is started with initial concentrations $X_{0}, P_{0}$ and $S_{0}$, $\left(P_{0}\right.$ is usually zero) and an initial volume of fermentation broth $V_{0}$. A constant feed flow $F$ supplies the reactor with an aqueous solution of fresh substrate (feed solution concentration: $S_{F}$ ). The expressions that correlate the instant volume of fermentation broth $V$ and the instant concentrations $X, P$ and $S$ with the time $t$ from the beginning of the process are given by Equations (1)-(4) [15]. It is noticed that, if $F=0$, the equations are still applicable and represent the batch mode. That is because they derive directly from the mass balance [4].

$$
\begin{gathered}
d V / d t=F \Rightarrow V=V_{0}+F \\
d X / d t=\mu X-F X / V \\
d P / d t=q_{p} X-F P / V
\end{gathered}
$$




$$
d S / d t=-\mu_{S} X+F\left(S_{F}-S\right) / V
$$

In this work, Saccharomyces cerevisiae ATCC 7754 cells were cultivated in yeast-peptone-dextrose (YPD) medium (comprised of yeast extract, peptone and dextrose with concentrations in proportions of 1:2:2) and $\mathrm{pH}$ of $6.5 \pm 0.2$, conditions adequate for their growth and that contain all the macro- and micronutrients needed. The cells were inoculated into a bioreactor with total volume of $2 \mathrm{~L}$. The other operational parameters were defined for keeping a constant proportion in relation to the fed-batch conditions previously applied to a total volume of $10 \mathrm{~L}$ [15]. Sucrose dissolved in deionized (DI) water $\left(S_{F}=30.0 \mathrm{~g} \cdot \mathrm{L}^{-1}\right.$, a condition of excess of substrate that prevents the growth inhibition related to lack of $S$ ) was fed with a flow of $6.67 \times 10^{-2} \mathrm{~L}^{-h^{-1}}$ using a peristaltic pump (MPS 380, Marte Científica, São Paulo, SP, Brazil) to a reactor with initial conditions $V_{0}=0.1 \mathrm{~L}, X_{0}=1.5 \mathrm{~g} \cdot \mathrm{L}^{-1}, S_{0}=10.0 \mathrm{~g} \cdot \mathrm{L}^{-1}, P_{0}=0$, and constant temperature of $33{ }^{\circ} \mathrm{C}$ to maximize the yield of this yeast [4]. The reactor design was chosen for obtaining a high ratio of surface area per liquid column height. Its surface was kept free to the atmospheric air and under magnetic stirring, guaranteeing the saturation of the liquid medium with air. Under the aerobic conditions, the cell reproduction is favored over the ethanol production (ethanol is mostly formed in anaerobioses) $[13,14]$. Therefore, the variation of refractive index due to the sucrose uptake by cells is not confused with the variation caused by high ethanol formation. Equations (1)-(4) were numerically solved (Euler method, step $0.1 \mathrm{~h}$, all differential equations simultaneously integrated) with the $\mu_{m}$ and $K_{M}$ values previously obtained for this same strain of microorganism operating under sucrose flow and cultivated in YPD under $33{ }^{\circ} \mathrm{C}\left(\mu_{m}=0.49 \mathrm{~h}^{-1}\right.$ and $\left.K_{M}=4.1 \mathrm{~g} \cdot \mathrm{L}^{-1}[4]\right)$. The other parameters were defined as the ones obtained in a previous work where a different strain of $S$. cerevisiae was cultivated in a complex medium similar to YPD (fermentation under fed-batch mode, $33^{\circ} \mathrm{C}$ ): $Y_{P / X}=2.660 \mathrm{~g} \cdot \mathrm{g}^{-1}, Y_{X / S}=0.2880 \mathrm{~g} \cdot \mathrm{g}^{-1}, m_{p}=0.010 \mathrm{~h}^{-1}$ and $m_{s}=0.290$ $\mathrm{h}^{-1}[15]$.

Besides that, solutions of sucrose in DI water with concentrations ranging from 2.5 to $100 \mathrm{~g} \cdot \mathrm{L}^{-1}$ (the concentration range commonly applied to industrial and laboratory fermentation processes [4]) had their refractive indexes (RI) evaluated with a MISCO PA 202 Refractometer (Palm Abbe, Cleveland, OH, USA). They were also tested with the fiber sensor for evaluating its sensitivity regarding $S$. Finally, the fed-batch reaction was online assessed over $3.5 \mathrm{~h}$ with the smartphone, and the results were compared to the refractometer and to the model predictions. The substrate instant concentrations were obtained by taking small samples of the fermentation broth for analysis of RI. All experiments and microbial cultures were performed in accordance with the Bioethical Committee of the University of Campinas, and with the declaration to Brazilian's Genetic Heritage Database (Register number: AD886EA).

\subsection{Sensing with the Smartphone}

The optical fiber sensor is based on the modulation of the power reflectance caused by differences in the refractive index of the liquid medium (originated by the consumption of sucrose by the cells [4]). Light is launch by a LED source (that may be the smartphone's camera LED or an external source) through a multi-mode silica optical fiber (MMF) and it is directed by a $2 \times 1$ coupler to the liquid medium. When light reaches the fiber-liquid interface, part of it is transmitted and part is reflected, as given by the Fresnel law [4]. Finally, the reflected light is redirected by the coupler to the smartphone's camera, and a developed application acquires and processes the data. Correct positioning of the optical fibers in relation to the smartphone's camera and the isolation from the environmental light are crucial for a reliable reading. For this purpose, a smartphone case was developed with dimensions for fitting a Samsung Galaxy 9 Plus smartphone $(76 \mathrm{~mm} \times 83 \mathrm{~mm} \times 14$ mm, hardware setup: Snapdragon $8452.8 \mathrm{GHz}, 6 \mathrm{~GB}$ RAM, $12 \mathrm{MP}$ resolution camera). The case contains ports for connecting the optical fiber to the camera and LED. It was manufactured by a 3Dprinter Ultimaker 2+ Extended (Ultimaker BV, Utrecht, The Netherlands) using poly (ethyleneglycol) filament. The full setup for the operation and analysis of the fed-batch fermentation system is shown on Figure 1A and the 3D-printed case on Figure 1B. 


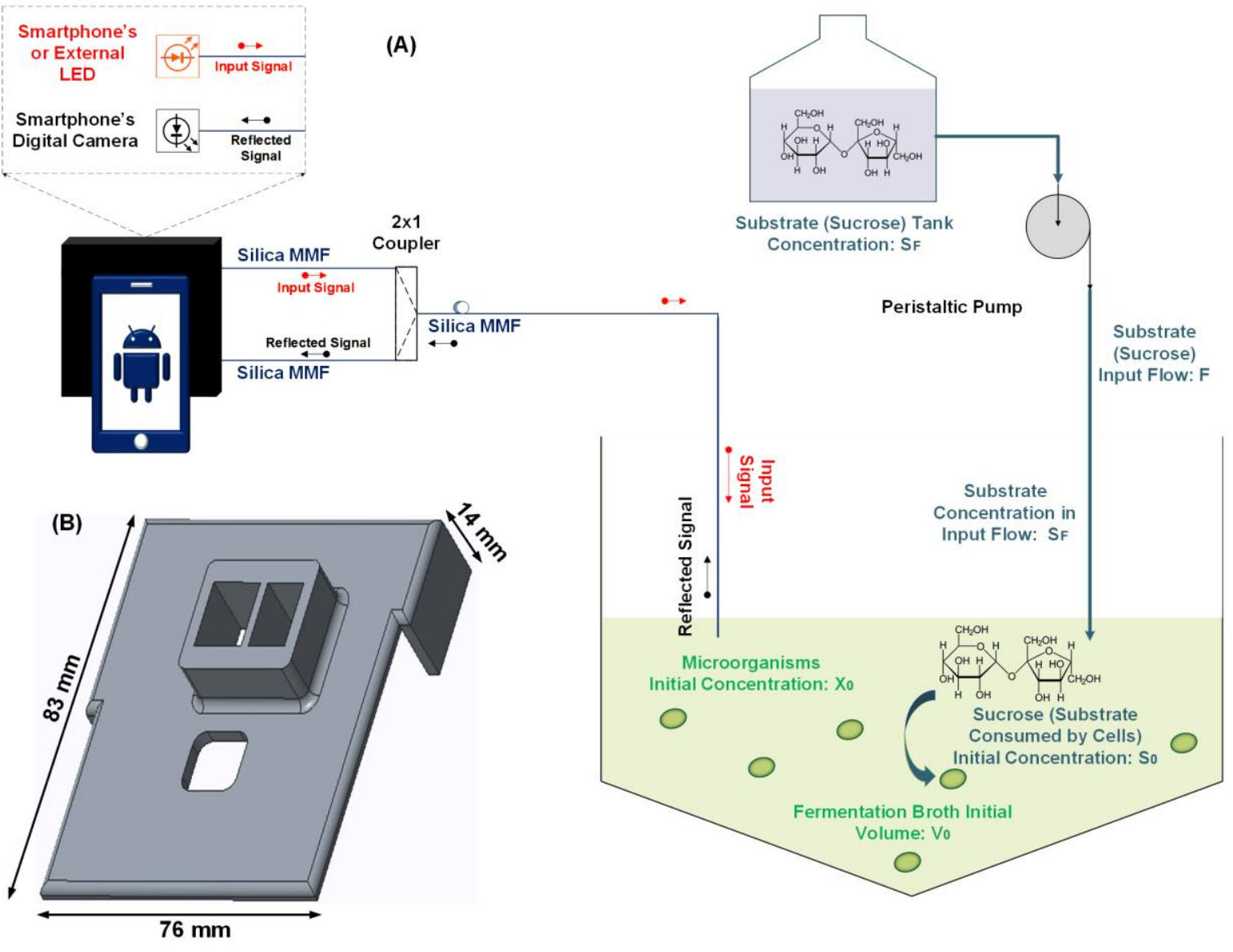

Figure 1. (A) Setup of the fed-batch fermentation and monitoring systems; (B) 3D-printed smartphone case.

An application for processing the intensity signals was developed in Android Studio. The operation is based on three steps: (i) setting image processing parameters; (ii) acquisition of the fiber output light; and (iii) analysis of intensity for each video frames. The application presents two operation modes: the first one evaluates the light intensity (pixel intensity) for a single image received from the camera (this mode is used for calibration and fiber alignment). The second mode performs the effective acquisition of data by recording a video, which is further analyzed frame-by-frame. The application reads the camera images, which consist of a dark background and an illuminated spot referent to the optical fiber termination, and calculates the average pixel luminous intensity $I_{m}$ on the selected region using the expression $I_{m}=\sum_{i=1}^{N} I_{w}\left(x_{i}, y_{i}\right) / N$. In this equation, the dark region is not considered for the calculus, $\mathrm{N}$ is the total number of pixels present on the region-of-interest, and $I_{w}\left(x_{i}, y_{i}\right)$ is the intensity level of the pixel in the coordinate $\left(x_{i}, y_{i}\right)$, given by the average of RGB channels. Finally, the processed light intensity data can be exported to a text file. Then, the normalized data are compared to the calibration curve for giving the value of $\mathrm{P}$.

\section{Results and Discussion}

Figure 2A shows both the correlation between RI and the sucrose concentrations ranging from 0 to $100 \mathrm{~g} \cdot \mathrm{L}^{-1}$, and the intensities detected by the smartphone-based sensor for the same solutions. Linear increases on $\mathrm{n}\left(n=1.3330+\left(1.4432 \times 10^{-4}\right) S, S\right.$ is the sucrose concentration in $\mathrm{g} \cdot \mathrm{L}^{-1}$, adjusted $\mathrm{R}^{2}=0.9971$ ), and on the average intensity $I_{m}$ were verified (normalized $I_{m}=85.8353 \mathrm{n}-63.6340, \mathrm{R}^{2}$ $=0.9344$ ). The sensitivity of the fiber sensor was calculated as the ratio of variation of the intensity with the refractive index, leading to a sensitivity of $85.83 \mathrm{RIU}^{-1}$ (refractive index units). The signal-tonoise ratios (SNRs) were evaluated as the relation $I_{m}{ }^{2} / \sigma^{2}$, where $\sigma$ is the signal standard variation: SNRs ranging from $2.25 \times 10^{2}$ to $2.4 \times 10^{5}$ were obtained. The fed-batch assessment is shown on Figure 
2B, where the mathematical model predictions (Equations (1)-(4)) are represented by a solid black line. Results obtained by the smartphone sensor corroborates with the refractometer and with the theoretical model analyses. In fermentation systems, relatively high deviations from the model are usually expected due to the uncertainties involved in representing different cells as a homogeneous population and to other different aspects of the bioprocess. There may be temporal evolution of the microorganism; adaption to small differences of substrate; small oscillations of temperature throughout the experiment; differences on shear stresses, etc. So, the constant recalculating of the adjusted parameters is highly suggested [4].
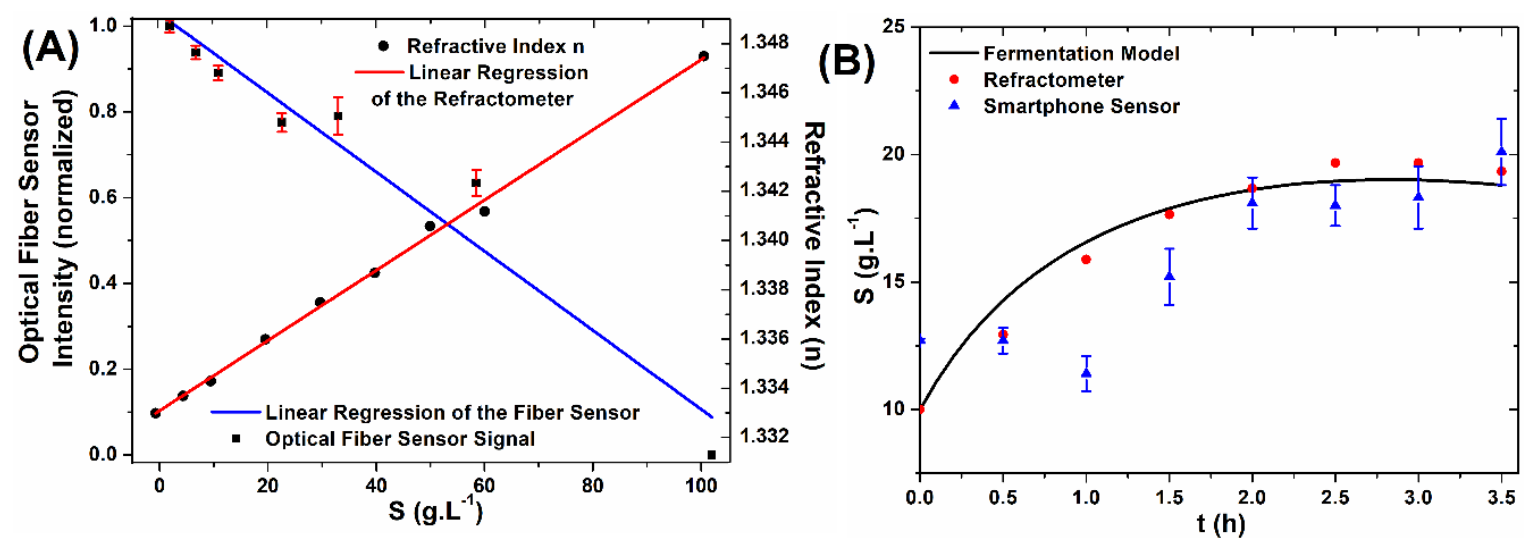

Figure 2. (A) Correlation between sucrose concentration $\boldsymbol{S}$ (red) and RI of the solution, and calibration of pixel intensity in relation to the RI (blue); (B) fed-batch fermentation results.

The refractometer presents a high resolution of $1 \times 10^{-4}$ RIU but is also a high-cost equipment that requires sample collecting throughout the experiment, in contrast to the smartphone-based fiber sensor that is available for online monitoring of the fermentation broth. Finally, it is important to notice that the other model equations (not shown on Figure 2) predicted a final concentration of $P$ (ethanol) of only $3.51 \mathrm{~g} \cdot \mathrm{L}^{-1}, 5.36$ times lower than the predicted concentration of sucrose $S(18.80$ $\left.\mathrm{g} \cdot \mathrm{L}^{-1}\right)$. Considering that the solution has approximately the same density as water under $33^{\circ} \mathrm{C}(994.8$ $\left.\mathrm{kg} . \mathrm{m}^{-3}\right), P$ corresponds to the mass percent of approximately $0.35 \%$. It is known that a fraction of $0.50 \%$ enhances the refractive index of a hydro-alcoholic solution to only 1.3334 [16]. This is the same effect of a solution containing concentrations as low as $2.5 \mathrm{~g} \cdot \mathrm{L}^{-1}$ of sucrose. Therefore, the ethanol effect on the RI can be indeed neglected.

\section{Conclusions}

The design of a portable and low-cost smartphone-based optical fiber sensor for the monitoring of bioreactors was demonstrated, providing a sensitivity of $85.83 \mathrm{RIU}^{-1}$ and a reliable assessment of the fermentation process. Due to the limited frequency of data collecting by the camera, the system is not capable of performing a quasi-elastic light scattering analysis like the sensor described in [4], where the direct evaluation of the biomass concentration was demonstrated. On the other hand, its production costs are quite inferior, it can be manufactured on-site by 3D-printing, and it can be easily integrated to an industrial line. This last characteristic is of major importance for the Industry 4.0, and such integration is not possible neither for the microfluidic systems nor the traditional assessment methods. Future works will focus on enhancing the sensitivity of the sensor and to test it under higher fermentation scales for the evaluation of its performance in analyzing more complexes systems. The smartphone processing will be also tested for the interrogation of special fibers and chemical sensors under development.

Author Contributions: M.C.P.S. and T.D.C. wrote the paper, prepared the yeast and the bioreaction system, and performed the data analysis, P.M.L. and M.d.S.R. performed the experiments; G.S.R. designed and fabricated the case; E.F. conceived the fiber sensor and provided funding. 
Funding: This research was funded by São Paulo Research Foundation (FAPESP) under grants 2017/20445-8 and 2019/22554-4, CNPq and Capes (Finance Code-001).

Acknowledgments: Authors thank Prof. Lucimara Gaziola de la Torre (School of Chemical Engineering FEQ/UNICAMP) for the yeast strain (S. cerevisiae ATCC 7754), and Prof. Cristiano Monteiro de Barros Cordeiro (Institute of Physics - IFGW/UNICAMP) for the handheld refractometer and 3D-printing equipment.

Conflicts of Interest: The authors declare no conflict of interest.

\section{References}

1. Pan, M.; Sikorski, J.; Kastner, C.A.; Akroyd, J.; Mosbach, S.; Lau, R.; Kraft, M. Energy Procedia 2015, 75, 15361541, doi:10.1016/j.egypro.2015.07.313.

2. Reis, M.S.; Kenett, R. AIChE J. 2018, 64, 3868-3881, doi:10.1002/aic.16203.

3. Ju, H.; Kandimalla, V.K. Electrochemical Sensors, Biosensors and Their Biomedical Applications; Zhang, X., Ju, H., Wang, J., Eds.; Elsevier: Amsterdam, The Netherlands, 2008.

4. Soares, M.C.P.; Vit, F.F.; Suzuki, C.K.; de la Torre, L.G.; Fujiwara, E. Perfusion Microfermentor Integrated into a Fiber Optic Quasi-Elastic Light Scattering Sensor for Fast Screening of Microbial Growth Parameters. Sensors 2019, 19, 2493, doi:10.3390/s19112493.

5. Li, X.; Nguyen, L.V.; Zhao, Y.; Ebendorff-Heidepriem, H.; Warren-Smith, S.C. Sens. Actuators B 2018, 269, 103-109, doi:10.1016/j.snb.2018.04.165.

6. Gong, C.; Gong, Y.; Chen, Q.; Rao, Y.-J.; Peng, G.-D.; Fan, X. Lab Chip 2017, 17, 3431-3436, doi:10.1039/C7LC00708F.

7. Basso, L.C.; Basso, T.O.; Rocha, S.N. Biofuel Production. Recent Developments and Prospects; Bernardes, M.A.S., Ed.; IntechOpen: Rijeka, Croatia, 2011; doi:10.5772/17047.

8. Hasenfratz, D.; Saukh, O.; Sturzenegger, S.; Thiele, L. In Proceedings of the 2nd International Workshop on Mobile Sensing, 2012.

9. Guo, J. Anal. Chem. 2016, 88, 11986-11989, doi:10.1021/acs.analchem.6b04345.

10. Dutta, S.; Sarma, D.; Nath, P. AIP Adv. 2015, 5, 057151, doi:10.1063/1.4921835.

11. Gunda, N.S.K.; Naicker, S.; Shinde, S.; Kimbahune, S.; Shrivastava, S.; Mitra, S. Anal. Methods 2014, 6, 62366246, doi:10.1039/C4AY01245C.

12. Michelini, E.; Calabretta, M.M.; Cevenini, L.; Lopreside, A.; Southworth, T.; Fontaine, D.M.; Simoni, P.; Branchini, B.R.; Roda, A. Biosens. Bioelectron. 2019, 123, 269-277, doi:10.1016/j.bios.2018.09.012.

13. Bailey, J.; Ollis, D. Biochemical Engineering Fundamentals; McGraw-Hill: New York City, NY, USA, 1986.

14. Doran, P. Bioprocess Engineering Principles, 2nd ed.; Elsevier: Amsterdam, The Netherlands, 2013.

15. Soares, M.C.P.; Luz, G.F.; Costa, A.C.; Gomes, M.K.; Mendes, B.F.; Torre, L.G.; Fujiwara, E. Saccharomyces cerevisiae Fed-Batch Fermentation and Artificial Intelligence Method for Adjusting Model Parameters to Experimental Data. Blucher Chem. Eng. Proc. 2018, 1, 2010-2014, doi:10.5151/cobeq2018-PT.0532.

16. Concentrative Properties of Aqueous Solutions: Density, Refractive Index, Freezing Point Depression, and Viscosity. In CRC Handbook of Chemistry and Physics, 100th ed.; Rumble, J., Ed.; CRC Press (Taylor and Francis Group): Boca Raton, FL, USA, 2019.

Publisher's Note: MDPI stays neutral with regard to jurisdictional claims in published maps and institutional affiliations.

2020 by the authors. Licensee MDPI, Basel, Switzerland. This article is an open access article distributed under the terms and conditions of the Creative Commons Attribution (CC BY) license (http://creativecommons.org/licenses/by/4.0/). 